\title{
On forced and free atmospheric oscillations near the 27-day periodicity
}

\author{
Ansgar Schanz ${ }^{*}$, Klemens Hocke and Niklaus Kämpfer
}

\begin{abstract}
The Whole Atmosphere Community Climate Model was used to investigate the influences of solar fluctuations on zonal wind oscillations. Two simulations were conducted with short-term solar forcing ( $<35$ days) on and off. We found that a 27-day wave is an inherent feature of the atmosphere when the short-term solar forcing is inactive. This internal 27-day oscillation comes along with other periods of the extra-long period wave band (20-40 days) and cannot be linked to the Sun's rotation period. When the short-term solar variability is part of the forcing, including the solar 27-day periodicity, it affects a wide range of the spectrum of zonal wind. At mid-latitudes, a 10-day wave emerges by the short-term solar forcing, which suggests that indirect and nonlinear interactions are involved. Solar short-term variability seems to generate atmospheric perturbations that interact with modes of the internal wave spectrum or the background mean flow. A robust and clear solar interpretation of these wind oscillations is challenging. However, dynamical responses to short-term solar variability exist and need further investigation.
\end{abstract}

Keywords: Solar forcing, Solar variability, Planetary waves, Zonal wind

\section{Background}

The radiation emitted by the Sun is not constant and shows modulations at different timescales. One example is the apparent 11-year solar cycle. At shorter timescales, a 27-day periodicity has been observed, which originates from anisotropic activities at the surface of the rotating Sun. Figure 1a displays the Lyman- $\alpha$ and 205-nm fluxes during the ascending solar cycle 24. Both wavelengths show a distinct 27-day modulation that is connected to the Carrington rotation period of the Sun. The shortterm ultraviolet (UV) flux variability can reach up to $25 \%$ for the active Sun ( $2 \%$ for the quiet Sun) and decreases towards higher wavelengths as shown in Fig. 1b.

These solar UV fluctuations induce intermittent responses in middle-atmospheric ozone and temperature (Hood and Jirikowic 1991; Chen et al. 1997; Dikty et al. 2010). Presumably, there also exist dynamical responses in terms of atmospheric waves, which usually follow steady forcing mechanisms or originate from shear flow instabilities and random wave excitation. At longer

*Correspondence: ansgar.schanz@bluewin.ch Institute of Applied Physics, Universität Bern, Sidlerstrasse, 3012 Bern, Switzerland periods, here, called extra-long periods (20-40 days), the solar 27-day periodicity is considered to contribute to excitations of Rossby waves (Krivolutsky et al. 2003). For instance, a solar modulation of extra-long wind periods was suggested by Luo et al. (2001) who found a weak positive correlation for interannual dynamics in regard to the 11-year solar cycle in the mesosphere.

The influence of solar variability is often described by efficiencies, i.e. to what extent an observable responds to an external variability. In this context, further hints of short-term solar influences on dynamics involve the high efficiencies of observed waves by UV flux variability (Hood and Jirikowic 1991; Krivolutsky et al. 2003). In pure photochemical calculations, the heating rate efficiency accounts for the $0.1 \mathrm{~K}$ per per cent increase of the 205-nm flux, which is two times less than that observed (Hood 1987). The apparent discrepancy has been related to a possible dynamical effect of short-term solar variability. Furthermore, Gruzdev et al. (2009) reported a dynamical response in the context of attenuated 16- to 20-day ozone waves in mid-latitudinal winter when the 27-day forcing was inactive. They presumed a nonlinear interaction of the 27-day signal with intrinsic 

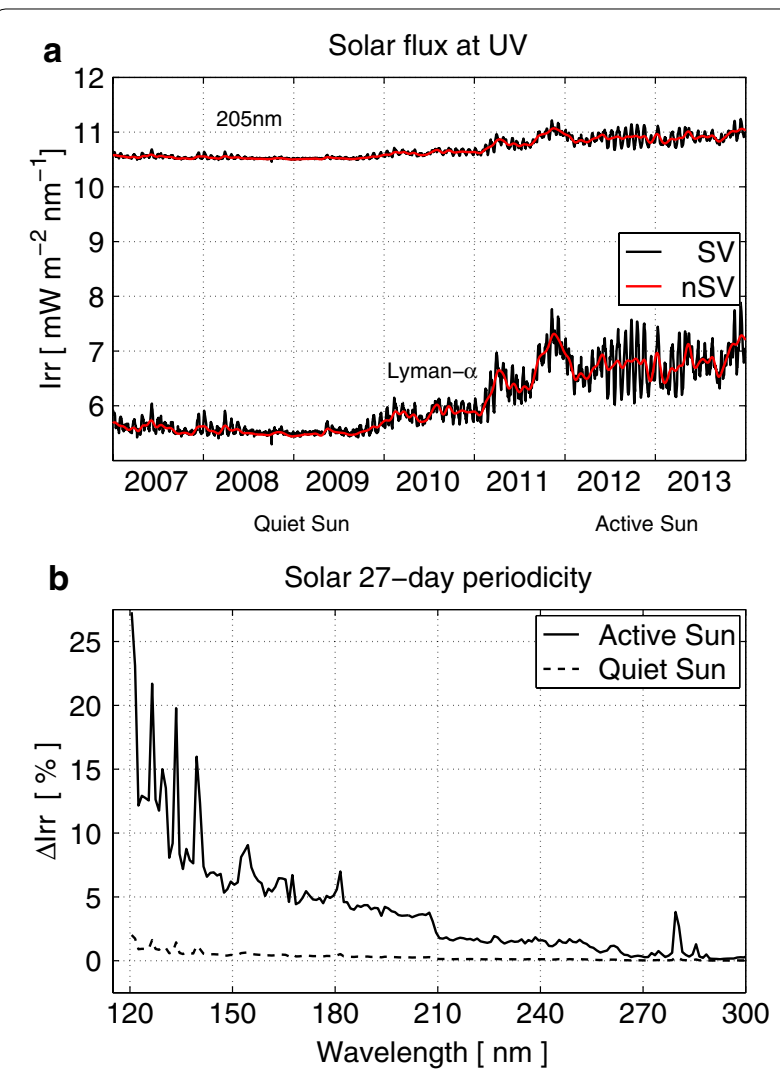

Fig. 1 Solar variability and 27-day periodicity. a The fluxes at Lyman-a $(121.6 \mathrm{~nm})$ and $205 \mathrm{~nm}$ during the ascending solar cycle 24 at 1 a.u. The Sun was quiet in 2008/2009 and shows increasing activity until 2014. b The relative flux variations at the 27-day periodicity for quiet and active Sun conditions. The presented solar data measured by the SORCE experiment (Lean et al. 2005) (black) and the derived low-pass-filtered fluxes (red) were used for the SV- and nSVsimulation, respectively

atmospheric variations, which may have changed the oscillation properties.

In this study, we calculate the dynamic responses for solar forcings with and without short-term solar variability by means of the Whole Atmosphere Community Climate Model (WACCM). One goal was to learn about the influences of the short-term solar variability on the zonal wind spectrum at extra-long periods. There was also an interest in examining how the atmospheric zonal wind spectrum changes when short-term solar variability is inactive. This will help with interpretations of wind spectra with respect to solar UV variability. Such middle-atmospheric wind spectra are now accessible by the new wind radiometer (WIRA) (Rüfenacht et al. 2012). These continuously measured wind profiles are unique since they sense wind directly and bridge the observation gap between 35 and $70 \mathrm{~km}$. Further, we are interested in learning how internal and forced oscillations accentuate in the spectrum at different atmospheric layers.
The letter is organized as follows: in section "Model data and methods" the model, simulations, and analysis methods are described. Section "Results and discussion" shows the simulation results of the WACCM for forcings with and without short-term solar variability. The conclusions give a summary of the findings and a brief outlook of future research directions.

\section{Model data and methods \\ Atmospheric model}

For this study, we used simulations of the WACCM. The model ranges from the Earth's surface to a model top at $10^{-6} \mathrm{hPa} / \sim 140 \mathrm{~km}$. The chemical representation of the stratosphere was based on the Model of Ozone and Related Tracers (MOZART v3) (Kinnison et al. 2007), which calculates the budgets of 59 atmospheric species.

Dynamics were simulated without the influences of data assimilation or dynamical nudging. The WACCM models the key coupling processes of the atmospheric layers, such as orographic and nonorographic gravity waves, mixing, sudden stratospheric warming events, strong convection cells, tides, and radiative coupling (Richter et al. 2008).

\section{Model simulations}

The numerical simulations were carried out with a spatial resolution of $4^{\circ}$ latitude by $5^{\circ}$ longitude and 66 layers on hybrid-pressure coordinates. The stratospheric photochemistry was coupled every $30 \mathrm{~min}$ to the model dynamics. The output has a temporal resolution of $6 \mathrm{~h}$.

We conducted simulations with solar irradiance data for the year 2013, which was almost at solar maximum conditions with strong UV variability. During this year, the model employed daily spectral solar irradiance (SSI) and total solar irradiance (TSI) data from the instruments used for the Solar Radiation and Climate Experiment (SORCE) (Lean et al. 2005). The absolute total irradiance was scaled to the PMOD composite (Fröhlich and Lean 1998; Fröhlich 2006) by a factor of 0.9965. The SSI data have a spectral resolution of $1 \mathrm{~nm}$ with a maximum uncertainty of approximately $0.15 \%$ in the UV range. The TSI data have an uncertainty of less than $0.05 \%$. The SORCE TSI and SSI data describe solar variability without the proxy of the $10.7-\mathrm{cm}$ radio flux.

Two identical simulations were conducted over a period of 1 year except that one lacked solar forcing. The first experiment was fed by the daily solar input without short-term variability. The SSI and TSI were low-pass filtered to eliminate all periods shorter than 35 days including the solar 27-day periodicity. The filter coefficients were set up with a Hamming window with a window length of three times the cut-off period. This ensured that the filter would have a fast response to the 
solar input data. The filter was applied to all wavelengths in the band from 120 to $10,000 \mathrm{~nm}$ and the TSI data. The second experiment was conducted with the daily solar input without filtering. Hereafter, the two experiments are referred to as the response to solar input with shortterm variability (SV) and without (nSV).

\section{Analysis methods}

The spectral analysis was performed by means of a zerophase finite impulse response (FIR) filter. The FIR filter was nonrecursive, and the filter coefficients were based on a Hamming window. The fast response to the time series was achieved by sampling time windows over three times the central period $T_{p}$. The cut-off frequencies of the band pass were $f_{c}=f_{p} \pm 10 \% f_{p}$, where $f_{p}$ represents the central frequency.

The envelopes (amplitudes) of zonal wind $\Delta u$ were calculated for periods from 5 to 40 days in $0.3 \overline{3}$-day steps. Further, $\Delta u$ was deduced for all latitudes by averaging the envelopes of the grid points of each circle of latitude (longitudinal steps of $20^{\circ}$ ).

\section{Results and discussion}

The atmospheric response to forcing without short-term variability (nSV-simulation)

From a first deduction of the nSV-simulation results without short-term solar forcing, the findings suggest that the 27-day oscillation in zonal wind is an inherent feature of the wave spectrum of the Earth's atmosphere. Moreover, in mid-latitudes such 27-day oscillations can be the dominant period of the extra-long period wave band. These surprising wave features occur in the nSVsimulation at different atmospheric layers and seasons.

Figure $2 \mathrm{a}, \mathrm{b}$ gives an overview of the zonal wind envelopes $\Delta u$ of the 27-day oscillations according to the nSV-simulation. Substantial 27-day variability resides at mid-latitudes in the upper troposphere lower stratosphere (UTLS) region $(6 \mathrm{~m} / \mathrm{s})$ and in the upper mesosphere $(10 \mathrm{~m} / \mathrm{s})$. Further, the quiet Arctic and Antarctic summer stratosphere shows weaker oscillations of the 27-day wave. Increased 27-day wave activity at mid-latitudes in the winter UTLS or mesosphere region seems comprehensible since the winter hemisphere is dynamically unstable compared to the summer hemisphere.

Further, Fig. 2 presents a wave spectrum at mid-latitudes $\left(42^{\circ} \mathrm{N}\right)$ from the surface to $0.01 \mathrm{hPa}$. These figures are separated into summer (c) and winter (d) periods. The 27-day period is dominant during winter in the UTLS region as shown in Fig. 2d (lower panel). Additionally, a clear 27-day zonal wind periodicity can be observed in the upper mesospheric summer. However, there is no dominant 27-day period in the middle and upper stratosphere at mid-latitudes.
The nSV-simulation of the fully coupled chemistry climate model WACCM demonstrates the important result that the 27-day oscillation in dynamics is not necessarily a response to the 27-day periodicity of the Sun. The occurring zonal wind oscillations are intriguing and might be explained as follows. Dynamics are driven by many different influences and show various intermittent phenomena, which propagate, interfere, dissipate, attenuate, or generate new waves. Similarly, earlier studies on ozone showed a 27-day signal when the solar shortterm forcing was inactive (Gruzdev et al. 2009), which could have originated from the same internal sources via dynamics. These findings indicate how challenging solar interpretation of zonal wind oscillations can be.

A potential source of atmospheric 27-day perturbations is the gravitational pull of the moon. Lunar influences such as the semidiurnal tide have been studied with the WACCM by adding terms to the meridional and zonal momentum equations (Pedatella et al. 2012). The present simulations are not affected by these disturbing lunar influences since are not comprised of lunar forcing or ocean tides. Moreover, such lunar influences are stronger in the mesosphere and lower thermosphere region and are small below $0.01 \mathrm{hPa}$ (Pedatella et al. 2012).

\section{The atmospheric response to forcing with short-term variability (SV-simulation)}

The simulation experiment was repeated with inclusion of short-term fluctuations of the solar input. The SV-simulation shows features of a response to the solar 27-day periodicity. Nevertheless, other features emerge in the extra-long period wave band spectrum and at shorter wavelengths such as a 10-day wave.

Figure 3a, b shows $\Delta u$ at $42^{\circ} \mathrm{N}$ from the SV-simulation in a similar manner as for the nSV-simulation. There exist interesting differences in the zonal wind spectra. For instance, in mesospheric summer, the short-term solar variability induces a strong 10-day oscillation (cf. Figs. 2c, 3a). This 10-day oscillation appears as a dominant period in the mesospheric spectrum. In stratospheric summer, from 1 to $10 \mathrm{hPa}$, the 27-day period is stronger than that for the nSV-simulation but not dominant. A further noticeable feature appears in the UTLS region at approximately $300 \mathrm{hPa}$. The clearly dominant 27-day peak of the nSV-simulation in winter is shifted towards the 35-day period (cf. Figs. 2d, 3b). To what extent this feature is connected to the low-pass cut-off at 35 days is unclear. Finally, in mesospheric winter, the periods of the extra-long period wave band are weaker compared to the nSV-simulation.

In this brief study we revealed that short-term solar forcing affects a wide range of the zonal wind spectrum at mid-latitudes. The clear 27-day signals at the UTLS 

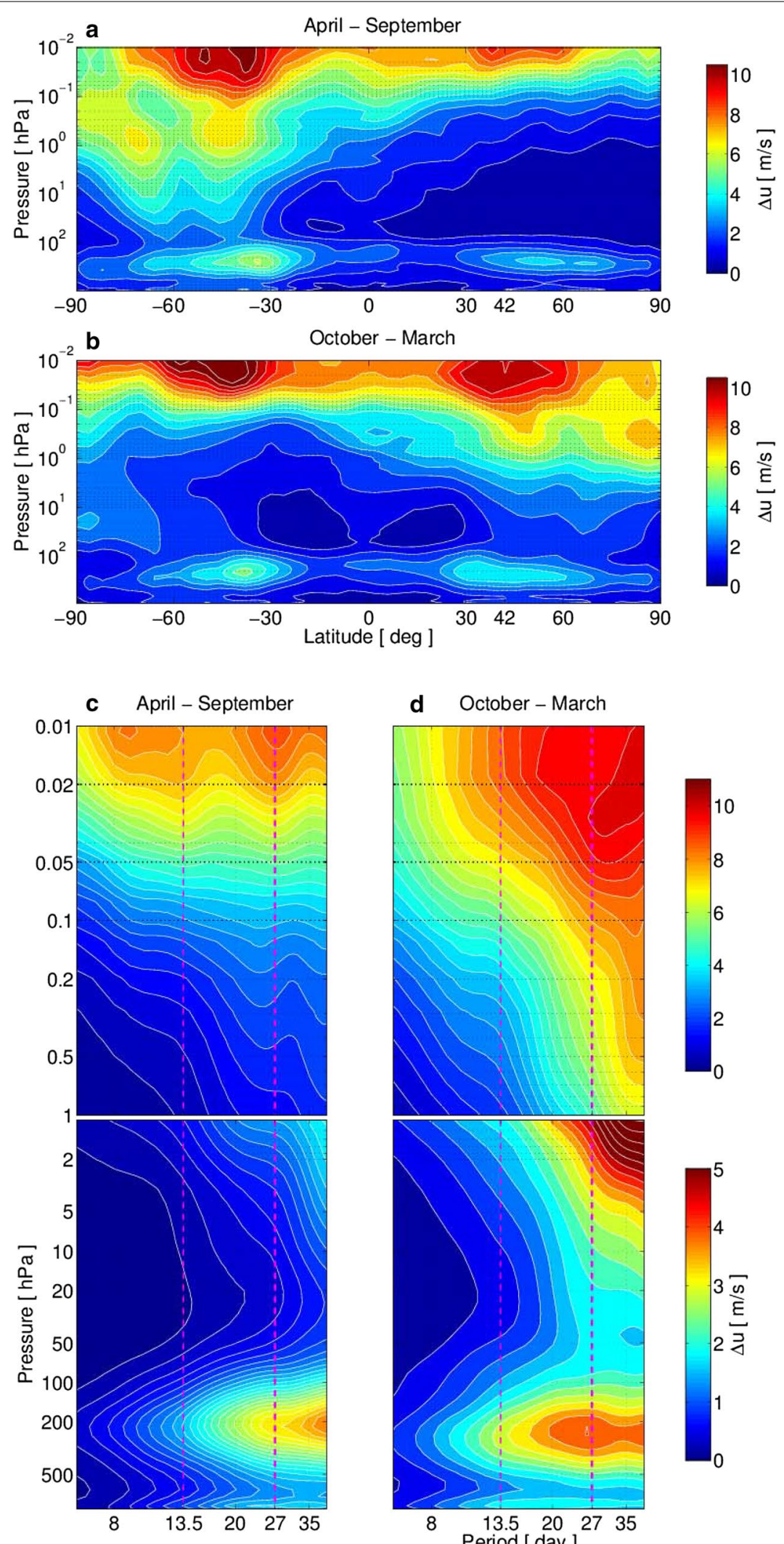

d October - March
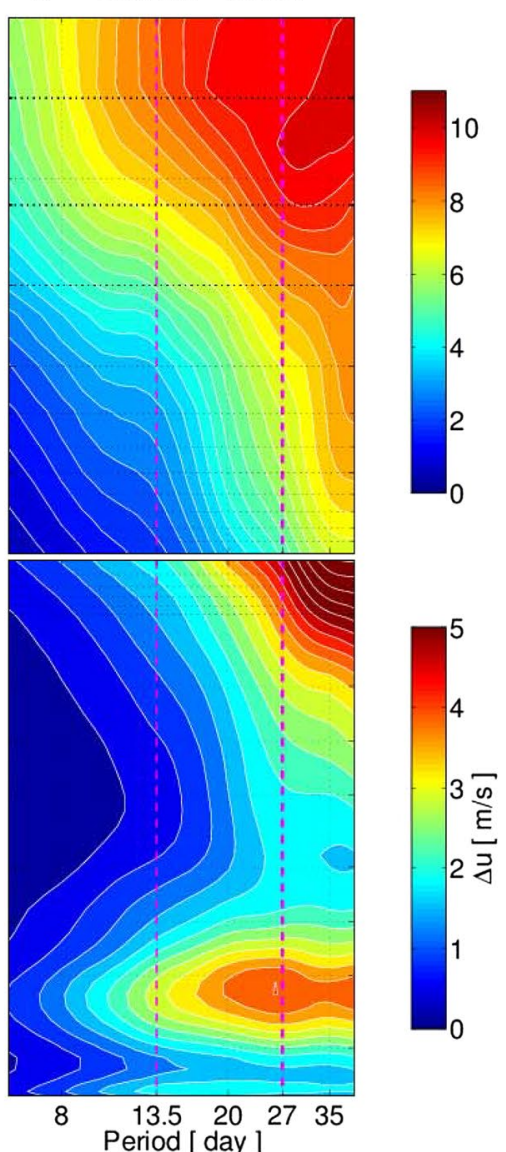
(See figure on previous page.)

Fig. 2 Zonal wind oscillation (nSV-simulation). Zonal wind envelopes $\Delta u$ for the 27-day oscillation from the nSV-simulation without short-term solar forcing. The upper two panels show $\Delta$ u over latitude for summer (a) and winter (b) periods. Strong activity of the 27-day oscillation occurred at mid-latitudes in the UTLS region and in the mesosphere. The lower panels show $\Delta u$ at $42^{\circ} \mathrm{N}$ in the mesosphere and in the stratosphere for summer (c) and winter (d) periods. The spectra of mesospheric summer and UTLS winter show clear 27-day signals in the nSV-simulation without short-term solar forcing. The magenta dashed lines refer to the solar 27-day periodicity and its 13.5-day harmonic. Please mind the two different colour codes for the panels

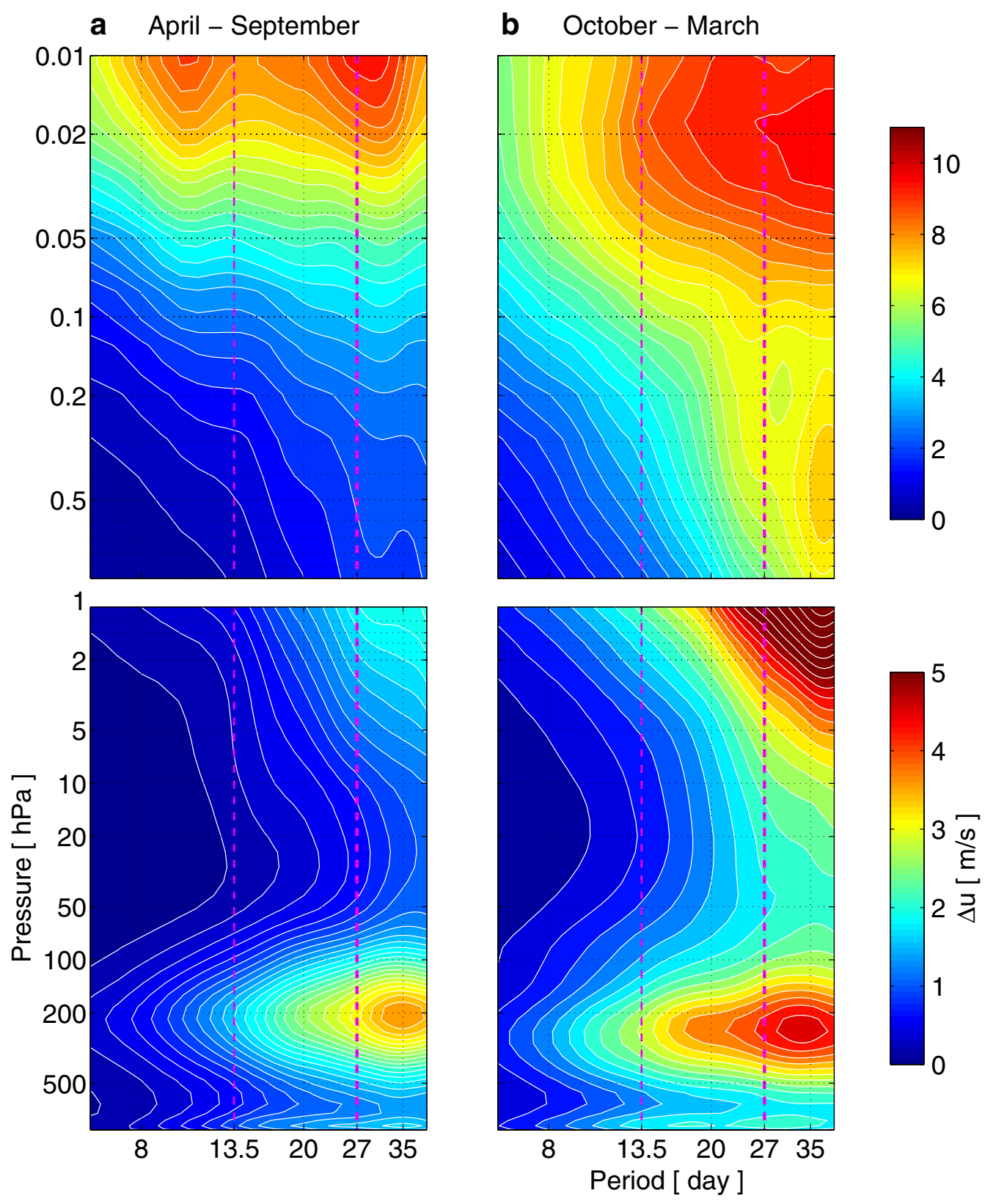

Fig. 3 Zonal wind spectrum at mid-latitudes (SV-simulation). Zonal wind envelopes $\Delta u$ at $42^{\circ} \mathrm{N}$ from the SV-simulation including the short-term solar forcing. The zonal wind envelopes are separated into panels for summer (a) and winter (b) periods. The spectrum of mesospheric summer shows a 10-day wave and a 35-day wave in the winter UTLS region when the short-term solar forcing is on. The magenta dashed lines refer to the solar 27-day periodicity and its 13.5-day harmonic. Please mind the two different colour codes for the panels 
region or the mesosphere in the nSV-simulation are more difficult to understand. Notably, there are internal modes at the same period as the solar 27-day periodicity which adds to the complexity. Additionally, the SV-simulation generated features near the 27-day period but also at other periods such as the enhancement of the 10-day wave. Presently, we cannot give a comprehensive explanation or a solar interpretation of these findings.

We assume that the solar-induced atmospheric perturbations are masked because of the interactions with the background zonal wind field and other atmospheric waves. An exception might be the mesosphere where the periods from 27 to 30 days during winter might reflect the unmasked solar-induced perturbation from the solar 27-day periodicity.

For further information, the reader is referred to the supplementary material associated with this manuscript in which we present spectra showing the difference in the zonal wind envelopes between the SV- and nSV-simulations for summer and winter conditions (Additional file 1).

\section{Conclusions and outlook}

We have presented a model experiment that focused on zonal wind oscillations and their connection to shortterm solar variability. From our two simulations with the WACCM and a case study at mid-latitudes, we were able to draw the following two main conclusions:

1. A 27-day zonal wind oscillation can be an inherent or even dominant period of the broad planetary wave spectrum at mid-latitudes.

2. The short-term solar variability influences the spectrum of planetary waves in a wide spectral range from a 10-day phenomenon to periods involving the extra-long period wave band. The perturbation of the solar 27-day periodicity in zonal wind is mostly masked.

Consequently, new questions have arisen. For instance, the reported 10-day wave phenomena might have contributions as a result of short-term solar perturbations. Further, our study did not discuss the phases of the induced atmospheric disturbances, which most likely lead to interesting interactions with inherent atmospheric waves, and this might explain some of the described features. A follow-on study aimed at reducing random components in the model by integrating over longer periods might be valuable. The WACCM-X model offers coupling to the ionosphere and also comprises also corpuscular solar input, which could further improve our knowledge on the atmospheric effects of short-term solar variability.
Finally, we think that modelling of the atmospheric planetary wave spectrum in the extra-long period wave band requires the full variability of solar input data.

\section{Additional file}

Additional file 1. Zonal wind spectrum at mid-latitudes (difference in the SV- and nSV-simulation). Difference spectra for zonal wind envelopes $\Delta u$ at $42^{\circ} \mathrm{N}$ from the SV-and nSV-simulation. The zonal wind envelopes are separated into panels for summer (a) and winter (b) periods.

\section{Authors' contributions}

AS performed the simulations and the data analysis. $\mathrm{KH}$ designed the filter algorithm. $\mathrm{KH}$ and NK contributed to the interpretation of the results. All authors read and approved the final manuscript.

\section{Acknowledgements}

The research leading to these results has received funding from the European Community's Seventh Framework Programme ([FP7/2007-2013]), Project NORS, under Grant Agreement No. 284421 (see Article II.30. of the Grant Agreement). We acknowledge the International Space Science Institute at Bern, Switzerland. Further, we would like to thank Han-Li Liu for consultations on the simulations and Rolf Rüfenacht for fruitful discussions. We further acknowledge Mark Whale for proof reading the manuscript.

\section{Competing interests}

The authors declare that they have no competing interests.

Received: 27 May 2015 Accepted: 3 May 2016

Published online: 03 June 2016

\section{References}

Chen L, London J, Brasseur G (1997) Middle atmospheric ozone and temperature responses to solar irradiance variations over 27-day periods. J Geophys Res 102(D25):29957-29979

Dikty S, Weber M, von Savigny C, Sonkaew T, Rozanov A, Burrows JP (2010) Modulations of the 27 day solar rotational signal in stratospheric ozone from scanning imaging absorption spectrometer for atmospheric cartography (SCIAMACHY) (2003-2008). J Geophys Res 115:D00115

Fröhlich C (2006) Solar irradiance variability since 1978, revision of the PMOD composite during solar cycle 21. Space Sci Rev 125:53-65

Fröhlich C, Lean J (1998) Total solar irradiance variations: the construction of a composite and its comparison with models. In: Correlated phenomena at the sun, in the heliosphere and in geospace. 31st ESLAB symposium ESA SP vol 415, pp 227-233

Gruzdev AN, Schmidt H, Brasseur GP (2009) The effect of the solar rotational irradiance variation on the middle and upper atmosphere calculated by a three-dimensional chemistry-climate model. Atmos Chem Phys 9:595-614

Hood LL (1987) Solar ultraviolet radiation induced variations in the stratosphere and mesosphere. J Geophys Res 92(D1):876-888

Hood LL, Jirikowic JL (1991) Stratospheric dynamical effects of solar ultraviolet variations: evidence from zonal mean ozone and temperature. J Geophys. Res 96(D4):7565-7577

Kinnison DE, Brasseur GP, Walters S, Garcia RR, Marsh DR, Sassi F, Harvey $V L$, Randall DE, Emmons L, Lamarque JF, Hess P, Orlando JJ, Tie XX, Randel W, Pan LL, Gettelman A, Granier C, Diehl T, Niemeier U, Simmons A (2007) Sensitivity of chemical tracers to meteorological parameters in the MOZART-3 chemical transport model. J Geophys Res 112:D20302

Krivolutsky AA, Kiryushov VM, Vargin PN (2003) Generation of wave motions in the middle atmosphere induced by variations of the solar ultraviolet 
radiation flux (based on the UARS satellite data). Int J Geomagn Aeron 3(3):267-279

Lean J, Rottman G, Harder J, Kopp G (2005) SORCE contributions to new understanding of global change and solar variability. Solar Phys 230:27-53

Luo Y, Manson AH, Meek CE, Igarashi K, Jacobi C (2001) Extra long period (20-40 day) oscillations in the mesospheric and lower thermospheric winds: observations in Canada, Europe and Japan, and considerations of possible solar influences 63:835-852
Pedatella NM, Liu HL, Richmond AD (2012) Atmospheric semidiurnal lunar tide climatology simulated by the Whole Atmosphere Community Climate Model. J Geophys Res 117:A06327

Richter JH, Sassi F, Garcia RR, Matthes K, Fischer CA (2008) Dynamics of the middle atmosphere as simulated by the Whole Atmosphere Community Climate Model, version 3 (WACCM3). J Geophys Res 113:D08101

Rüfenacht R, Kämpfer N, Murk A (2012) First middle-atmospheric zonal wind profile measurements with a new ground-based microwave Dopplerspectro-radiometer. Atmos Meas Tech 5:2647-2649

\section{Submit your manuscript to a SpringerOpen ${ }^{\circ}$ journal and benefit from:}

- Convenient online submission

- Rigorous peer review

- Immediate publication on acceptance

- Open access: articles freely available online

- High visibility within the field

- Retaining the copyright to your article 